\title{
A unilateral field advantage for detecting repeated elements
}

\author{
Serena Jenelle Butcher and Patrick Cavanagh \\ Harvard University, Cambridge, Massachusetts
}

\begin{abstract}
Previous studies have shown that letter repetitions are detected more rapidly when presented bilaterally (one letter in each visual field) than when presented unilaterally (both in the same field) when subjects have to report matches independently of case or font (e.g., Aa). This pattern of results is referred to as a bilateral field advantage. Here, we present evidence of an opposite pattern of results for detecting repeated items when they are physically identical. In our repetition detection paradigm, subjects indicated whether there was a repetition of any two of four presented items, one in each quadrant of the visual field. Stimulus classes tested included letters, color, size, orientation, and motion paths. The subjects were significantly faster at detecting unilateral versus bilateral repetitions for four out of the five stimuli classes tested, with a trend in the same direction for the fifth. This unilateral field advantage suggests that low-level processes group physically identical items more efficiently within hemifields than across.
\end{abstract}

The perception of visual space seems continuous. When we open our eyes to view a scene, we effortlessly perceive a world of contiguous visual space. However, our processing of visual space is, in fact, divided. When a central point is fixated, the proximal stimulus on the retina is inverted and mirror reversed. Information on the right side of space in the right visual field (RVF) is transmitted to the left hemisphere of the brain, whereas information on the left side of space in the left visual field (LVF) is transmitted to the right hemisphere.

The division of processing with respect to visual fields affords the ability to examine the visual processes that can be preformed independently by each hemisphere prior to interhemispheric integration. One method for doing so is to present stimuli to either one or both visual fields and examine whether same-field or across-field presentation increments or decrements performance, as measured by reaction time (RT) and/or accuracy.

In previous studies, tasks in which presentation of stimuli to both visual fields enhances performance have been examined. This pattern of results is known as a $b i$ lateral field advantage. This advantage has been reported for tasks in which multiple elements must be compared to make a decision about abstract similarity. For example, subjects are faster to respond that two letters, mismatched in case or font, are matched in identity when they are presented bilaterally to both visual fields, versus unilaterally to the left or right field (see Figure 1; Banich \& Belger, 1990; Belger \& Banich, 1992; Lavidor \& Ellis, 2003; Ludwig, Jeeves, Norman, \& DeWitt, 1993).

These types of same/different tasks require the processing of stimuli to the level of letter name before a response is made. The bilateral field advantage suggests that at least some portion of the analysis leading to letter recognition may proceed independently in the left and right hemifields but must proceed sequentially when both letters are in one field. If this interpretation is correct, the results suggest a range of savings in processing time of about $10-80 \mathrm{msec}$ (Banich \& Belger, 1990; Belger \& Banich, 1992; Lavidor \& Ellis, 2003; Ludwig et al., 1993), due to the concurrent analyses in the two hemifields.

Here, we report evidence of an opposing pattern of results: a unilateral field advantage for detecting repetitions that consist of physical matches, rather than name matches of items that are not physically identical (e.g., Aa). In our repetition detection paradigm, stimuli were presented at the four corners of a virtual square. All four locations were filled on each trial, and the subject reported the presence or absence of a repeated element. Across the experiments, unilateral repetitions were detected more quickly than bilateral repetitions for the letter, color, size, and, to a lesser extent, orientation stimuli. Furthermore, we present evidence that a unilateral field advantage can arise from both moving and static repetitions.

There is prior evidence that unilateral visual field presentation affords an advantage in response time when the stimulus decision involves a perceptual (vs. abstract identity) match. Common paradigms for these experiments include presenting subjects with two different letters around fixation (one in each hemifield) and presenting a physically matching test letter below one of them.

For within-hemifield trials, the test letter matches the letter above in the same hemifield. In the between-hemifield case, the letter match is below a nonmatching letter in the op-

S. J. Butcher, sbutcher@andrew.cmu.edu 

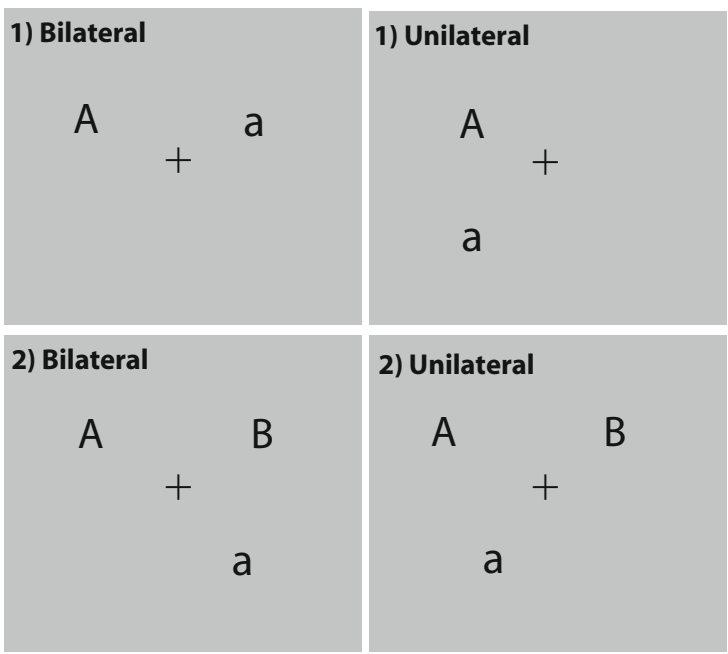

Figure 1. Two common paradigms that produce a bilateral field advantage pattern of results. Top panel: Subjects are faster (approximately 10-40 msec) to respond that two letters are the same in identity (as in this example) or different in identity when they are presented bilaterally (top left) versus unilaterally (top right) to either the left visual field or the right visual field (Ludwig et al., 1993). Bottom panel: In this paradigm, subjects are presented with two letters in the upper visual field, which never match in identity. After a brief interval, an additional letter is presented in the lower visual field. Subjects are faster (approximately $50-80 \mathrm{msec}$ ) to say that the lower letter is an identity match to an upper visual field letter when it appears bilaterally (bottom left) versus unilaterally (bottom right) (Banich \& Belger, 1990; Belger \& Banich, 1992; Lavidor \& Ellis, 2003).

posite hemifield (Banich \& Belger, 1990; Lavidor \& Ellis, 2003). However, the triangular arrangement of task stimuli creates a display in which within-hemifield letter matches are in closer proximity than across-hemifield matches; thus, physical distance cannot be ruled out as a factor in producing a unilateral advantage for perceptual matches. In our paradigm task, relevant stimuli were presented at the vertices of a square. Thus, equidistant within-hemifield and acrosshemifield perceptual matches could be directly compared.

Banich and colleagues have posited that within-field advantages occur when the computational complexity of a task is low (Banich, 1998a, 1998b; Weissman \& Banich, 2000). A bilateral field advantage occurs when the computational complexity of a task increases, suggesting that computationally complex tasks benefit from the processing power of two hemispheres afforded by bilateral visual presentations. We suggest that the unilateral field advantage reflects perceptual grouping, a task of low computational complexity. Furthermore, there is a cost associated when grouping must be coordinated between hemifields and across hemispheres.

We propose that the unilateral field advantage for detecting physically identical items arises because the physical repetition of adjacent items forms a salient group that can be detected by low-level processes. In particular, our results suggest that this low-level grouping operates more efficiently within versus across hemifields.

\section{GENERAL METHOD}

In five separate experiments, subjects searched for the repetition of an element in a visual search display. The first experiment involved letter elements, the second colors, the third size, the forth orientation, and the fifth repeated motion trajectories.

\section{Subjects}

Eight subjects were tested in each experiment. All the subjects reported normal or corrected-to-normal visual acuity and color vision.

\section{Apparatus}

All the stimuli were presented by a Macintosh computer on a 17-in. Apple Studio Display monitor at a resolution of $1,280 \times$ 1,024 and a refresh rate of $75 \mathrm{~Hz}$ with Visionshell software (www .visionshell.com).

\section{Procedure}

In all the experiments, the subjects' task was to determine whether two identical elements were present in a display of four items. On target-present trials, two out of four items were identical; on targetabsent trials, all four items were unique. The four search items always appeared in the same positions during the experiment. The four search positions formed the vertices of an invisible square around fixation.

Repeated elements could occur at any two positions constituting endpoints for a line segment on any one side of the square (top/ upper visual field, bottom/lower visual field, left/left visual field, and right/right visual field), but not at two points that corresponded to a diagonal bisecting the square. This manipulation ensured that the distance between bilaterally presented target items (i.e., repeated elements) was similar to the distance between unilaterally presented target items. Target position was selected randomly for each trial. All the target positions were used with the same frequency. All the search stimuli were presented on a uniform gray background.

In each experiment, the subjects pressed a key to initiate each trial. A fixation point was shown at the center of the screen for $100 \mathrm{msec}$. The start of a trial was preceded by a tone. The subjects indicated the presence or absence of a repeated element via the computer keyboard, pressing the "key for a repetition and the "A" key for no repetition. The subjects were instructed to respond as quickly as possible to the presence or absence of the target items, while maintaining fixation and minimizing errors.

\section{Data Analysis}

For all the experiments, a one-way repeated measures MANOVA with four levels (corresponding to the four possible configurations of repetitions) was performed on both the RT and the error data. For RT, only RTs for correct responses to target-present trials were analyzed. RTs greater than $3,000 \mathrm{msec}$ and less than $150 \mathrm{msec}$ were considered errors and were excluded (fewer than $0.06 \%$ of the trials were removed from each experiment). For error analyses, only misses (erroneous absent responses) were analyzed as a function of target position. Contrast analyses were used to examine RT and error differences as a function of target position and laterality.

\section{EXPERIMENT 1 Letter Repetition Search}

\section{Stimuli and Procedure}

The stimuli for the search task were uppercase letters in the Geneva font and subtended no more than $0.8^{\circ}$ (length) $\times 0.6^{\circ}$ (width) at the $57-\mathrm{cm}$ viewing distance. Target and distractor letters were selected randomly from the 26 letters on each trial; thus, the size measurement corresponded to the length and width of the largest letter. The subjects performed 20 practice and 300 experimental trials. A repeated letter was present on $67 \%$ of the trials. All the letter stimuli were presented at the vertices of an invisible $3^{\circ} \times 3^{\circ}$ square around fixation. 


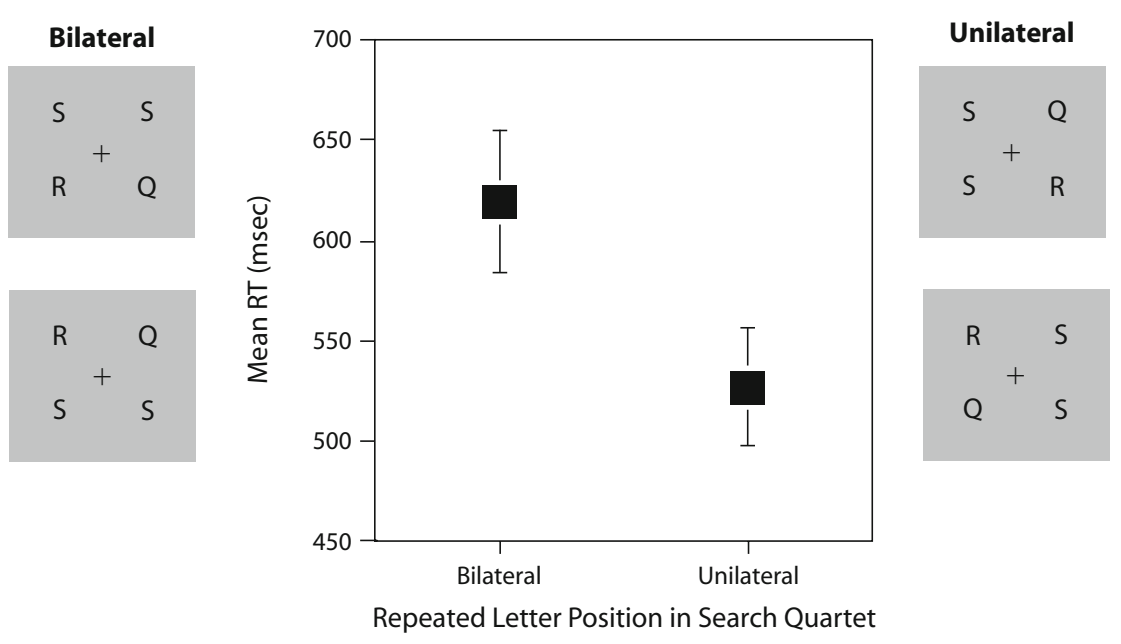

Figure 2. Target-present mean reaction times (RTs) for detecting bilateral and unilateral letter repetitions $(n=8)$ in Experiment 1. Unilateral targets were detected significantly more quickly than bilateral targets. Error bars indicate \pm 1 SEM.

\section{Results}

Figure 2 shows mean RT as a function of the targets' laterality. Bilateral corresponds to letter repetitions occurring in the top and bottom of the search quartet. Unilateral corresponds to letter repetitions occurring in the LVF and RVF. Unilateral repetitions were detected $92 \mathrm{msec}$ more quickly than bilateral repetitions $[F(1,7)=25.22, p<$ $.002]$. The target-absent mean RT was $715 \mathrm{msec}(S E M=$ $\pm 25 \mathrm{msec})$. The mean overall error rate was $6 \%$ (SEM $=$ $\pm 2 \%$ ). There was no evidence of a speed-accuracy tradeoff in detecting unilateral letter repetitions (mean unilateral misses $=2 \%, S E M= \pm 1 \%$; mean bilateral misses $=$ $4 \%, S E M= \pm 2 \%)$. The average false alarm rate was $2 \%$ $(S E M= \pm 1 \%)$.
Figure 3 shows mean RT as a function of each bilateral target position (left panel) and for each unilateral target position (right panel). There was no significant difference in RT for detecting repeated letters in the top versus the bottom of the letter quartet. Similarly, there was no difference in RT between the LVF and the RVF.

\section{Discussion}

The results of Experiment 1 demonstrate a unilateral field advantage for detecting physically identical repeated letters. The subjects were consistently faster to say that two letters were repeated when they were presented in the same visual field, regardless of whether the repetition occurred in the LVF or the RVF.
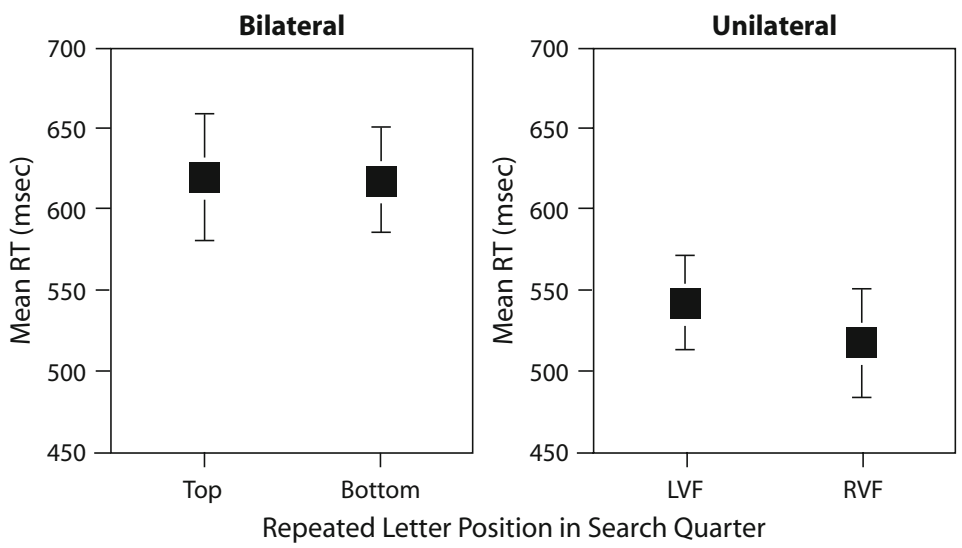

Figure 3. Left panel: Mean reaction times (RTs) as a function of repeated letter target position, top versus bottom, for bilateral targets in Experiment 1. Right panel: Mean RTs as a function of target position, left versus right, for unilateral targets $(n=8)$. For bilateral presentations (left panel), there was no significant difference in RTs between targets appearing in the upper versus the lower visual field. Similarly, for unilateral presentations (right panel), there was no significant difference in RTs for left-visual-field and right-visual-field targets. 
However, in this experiment, the unilateral field repetitions were confounded with vertical alignment. It may be the case that vertically aligned repetitions were easier to detect than horizontally aligned ones, regardless of whether the repetition occurred within or across visual fields.

To examine this question, we ran a control experiment in which the entire search quartet was presented either around fixation (similar to Experiment 1) or to the left or right of the vertical meridian. Quartets presented to the left fell entirely in the LVF, whereas quartets to the right fell in the RVF. This manipulation allowed us to examine whether the results of Experiment 1 indicated an advantage for detecting unilateral letter repetitions or whether the vertical alignment of elements produced the RT benefit.

\section{CONTROL EXPERIMENT 1.1 Is Vertical Alignment of Repeated Elements Special?}

\section{Stimuli and Procedure}

The stimuli and task in this control experiment were identical to those used in Experiment 1, with the following exceptions. The search quartet could be presented either around fixation (as in Experiment 1 ) or shifted horizontally $3^{\circ}$ to the left or right of fixation. The search quartet appeared an equal number of times around fixation and in the LVF and RVF. However, its position varied randomly from trial to trial. The subjects were instructed to maintain fixation at the central fixation point regardless of where the quartet appeared. The subjects preformed 20 practice and 300 real trials. A repeated letter was present on $67 \%$ of the trials.

\section{Results}

Figure 4 shows the mean RT for each search quartet as a function of whether the repeated letter target was horizontally or vertically aligned. In the central quartet, horizontal alignment corresponded to a bilateral field presentation and vertical alignment to a unilateral field presentation. For this central presentation, the mean RTs were $513 \mathrm{msec}$ for vertically aligned (unilateral) repetitions and $625 \mathrm{msec}$ for horizontally aligned (bilateral) repetitions. The 112-msec RT advantage was significant $[F(1,7)=31.02, p<.001$, two-tailed], replicating the result of the first experiment. Mean error in this quartet was $6 \%$. There was no evidence of a speed-accuracy trade-off (mean unilateral misses $=1 \%, S E M= \pm 1 \%$; mean bilateral misses $=7 \%, S E M= \pm 3 \%)$. The mean false alarm rate was $4 \%(S E M= \pm 2 \%)$.

For quartets presented entirely within the LVF and RVF, horizontally and vertically aligned repetitions were always within a single visual field. In the LVF, there was no difference between horizontal and vertical repetitions. The mean error for the LVF was $5 \%(S E M= \pm 2 \%)$. The mean false alarm rate for the LVF was $4 \%$ (SEM = $\pm 2 \%$ ). In the RVF, horizontal repetitions were detected $50 \mathrm{msec}$ more quickly than vertical ones $[F(1,7)=$ $33.58, p<.001$, two-tailed], reversing the effect seen for the central location. The mean error for the RVF was $5 \%(S E M= \pm 2 \%)$. The mean false alarm rate for the LVF was $2 \%(S E M= \pm 1 \%)$.

Vertical repetitions around fixation were detected more quickly than ones in the far left of the LVF quartet and the far right of the RVF quartet $[F(1,7)=32.66, p<.000$, two-tailed]. However, there was no difference in RTs between detecting vertical repetitions in the far left of the LVF and the far right of the RVF.

The target-absent mean RTs were $773 \mathrm{msec}$ for the LVF, $742 \mathrm{msec}$ for the center, and $770 \mathrm{msec}$ for the RVF. The mean error rate for all quartet locations was $6 \%(S E M= \pm 3 \%)$.

\section{Discussion}

The results of Control Experiment 1.1 indicated that there was no advantage of vertical alignment per se. Figure 4 illustrates that the main factor in determining how quickly the subjects found a repeated letter was whether the repetition occurred within or across visual fields. If vertical alignment were indeed special, the subjects would have been faster at detecting vertical repetitions in all quartet locations. However, the subjects were faster at detecting the vertical repetitions only for the central quar-
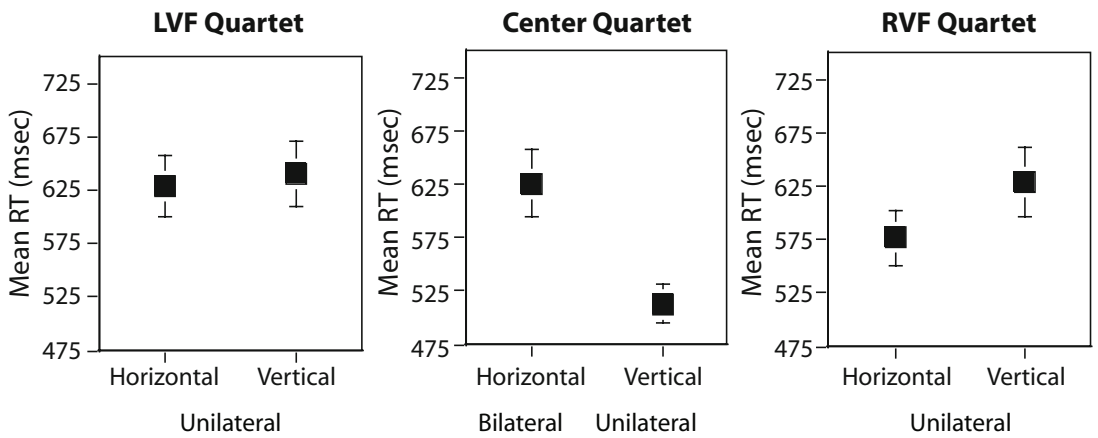

Repeated Letter Position in Search Quartet

Figure 4. Control Experiment 1.1: Mean reaction times (RTs) as a function of letter repetition orientation, horizontal and vertical for left visual field (LVF), center, and right visual field (RVF) search quartets. Vertical alignment of a repeated letter produced a unilateral field advantage only in the central quartet $(112.50 \mathrm{msec}, p<.001)$, in which this alignment corresponded to unilateral versus bilateral presentation. 
tet, and only there did the vertical alignment correspond to a unilateral field presentation.

When the entire display was shifted to the LVF or RVF, the subjects were slower, overall, in detecting repetitions, likely due to the greater eccentricity of the elements in the periphery. This is suggested by the RT advantage for detecting vertical targets around fixations versus in the periphery. There was no difference in detecting vertical repetitions that appeared in the far LVF versus the far RVF. There was no advantage for detecting vertically aligned letter repetitions. In the LVF, there was no difference between horizontal and vertical repetitions. In the RVF, horizontal repetitions showed an advantage.

Thus far, we have presented evidence for a unilateral field advantage for detecting repeated letters that cannot be explained by the vertical alignment of repeated elements. However, in both Experiment 1 and Control Experiment 1.1, the display was present until the subjects made a response. This left open the possibility that the subjects could fixate individual stimuli, making the RT advantage more difficult to interpret in terms of laterality. To address this concern, we ran a second control experiment in which the entire search display was presented for a single video frame $(15 \mathrm{msec})$. This presentation time was too brief for the subjects to move their fixation.

\section{CONTROL EXPERIMENT 1.2 Brief Presentation Letter Repetition Search}

\section{Stimuli and Procedure}

The stimuli and procedure were identical to those in Experiment 1 , with the following exceptions. The letter search display was presented for one video frame, $15 \mathrm{msec}$. The subjects preformed 20 practice and 400 real trials.

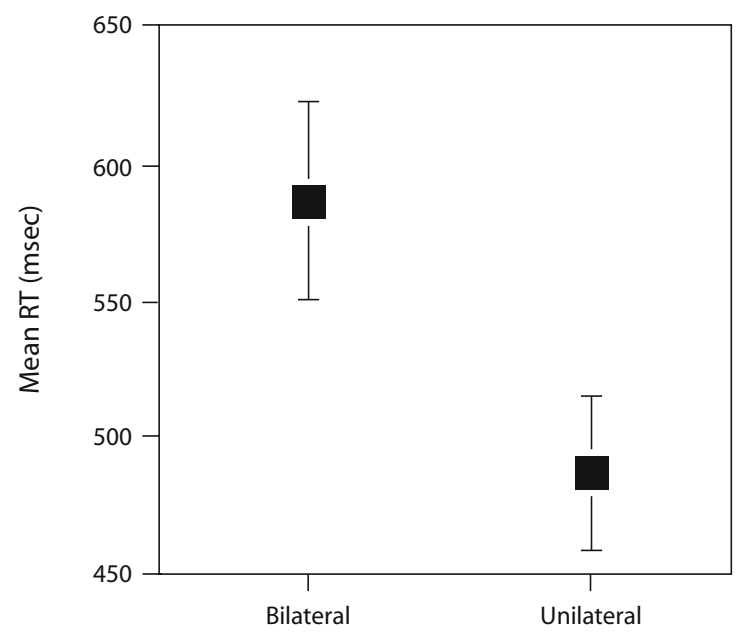

Repeated Letter Position in Quartet

Figure 5. Control Experiment 1.2: Target-present mean reaction times (RTs) as a function of repeated letters' laterality $(n=8)$. The search display was presented for $15 \mathrm{msec}$. Unilateral letter repetitions were detected significantly more quickly than bilateral repetitions $(p<.00)$. Error bars indicate \pm 1 SEM.

\section{Results}

Figure 5 shows mean RT as a function of the target repetition's laterality. Unilateral repetitions were detected 99 msec more quickly than bilateral repetitions $[F(1,7)=$ 109.87, $p<.00$, two-tailed]. The target-absent mean RT was $654 \mathrm{msec}$. The mean error was $9 \%(S E M= \pm 3 \%)$. There was no evidence of a speed-accuracy trade-off (mean unilateral misses $=5 \%, S E M= \pm 1 \%$; mean bilateral misses $=1 \%, S E M= \pm 2 \%)$. The mean false alarm rate was $2 \%(S E M= \pm 1 \%)$.

For unilateral letter repetitions, the targets in the RVF were detected more quickly than the LVF targets $[27 \mathrm{msec}$; $F(1,7)=12.26, p<.01$, two-tailed]. For bilateral repetitions, the upper visual field targets were detected more quickly than the lower visual targets $[120 \mathrm{msec} ; F(1,7)=$ $38.95, p<.00$, two-tailed]. Due to the significant difference in repetition detection for bilateral presentations, we ran an additional comparison between the upper visual field (faster bilateral position) and unilateral repetitions. Unilateral repetitions were detected significantly more quickly than repetitions in the upper visual field [ $46 \mathrm{msec}$; $F(1,7)=109.86, p<.000$, two-tailed].

\section{Discussion}

A significant unilateral field advantage again was found for detecting letter repetitions presented too briefly to allow the subjects to make saccades to individual elements. This suggests that eye movements are not a factor in the unilateral field advantage. However, the brief presentation paradigm produced an error rate higher than that in Experiment 1, where the stimulus remained on screen until the subjects responded.

In addition, for the category of bilateral presentations, there was a significant difference in performance between the upper and the lower visual field targets. However, unilateral targets were detected more quickly than those in the bilateral top position (faster bilateral position), suggesting that unilateral presentation of repetitions affords a distinct advantage over bilateral presentation, with regard to speed of detection. In the following experiments, we provide evidence that the unilateral field advantage extends to other stimulus classes, in addition to letters.

\section{EXPERIMENT 2 Color Repetition Search}

\section{Stimuli and Procedure}

Colors were presented as colored squares that subtend $1.5^{\circ} \times 1.5^{\circ}$ at a $57-\mathrm{cm}$ viewing distance. Colors for each trial were randomly selected from a set of six colors: red, blue, cyan, green, yellow, and purple. The color stimuli were presented at the vertices of an invisible $3^{\circ} \times$ $3^{\circ}$ square around fixation and remained on screen until the subjects responded. A repeated color target was present on $50 \%$ of the trials.

\section{Results}

Figure 6 shows mean RT as a function of whether the color repetition was presented unilaterally or bilaterally to the visual fields. Unilateral repetitions were detected $57 \mathrm{msec}$ more quickly than bilaterally presented ones $[F(1,7)=31.01, p<.001$, two-tailed]. The targetabsent mean RT was $595 \mathrm{msec}$. The mean error rate was 


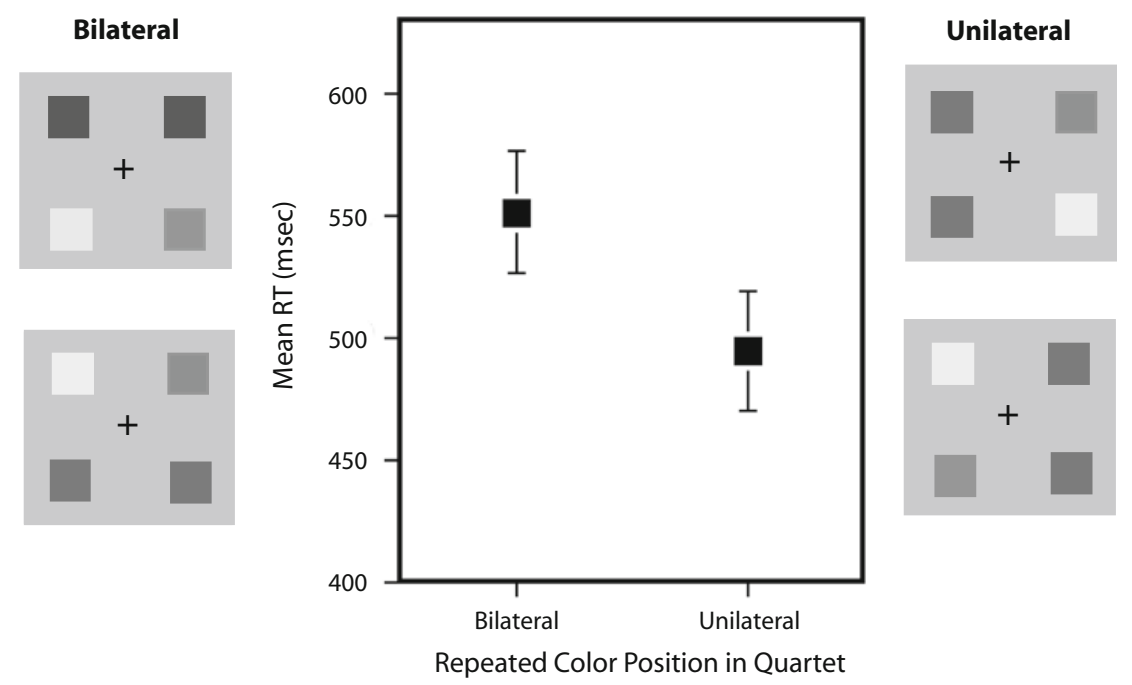

Figure 6. Experiment 2: Color repetition search. Target-present mean reaction times (RTs) as a function of bilateral versus unilateral repetition presentation $(n=8)$. Unilateral color repetitions were detected significantly more quickly than bilateral color repetitions.

$4 \%(S E M= \pm 1 \%)$. There was no evidence of a speedaccuracy trade-off (bilateral misses $=4 \%, S E M= \pm 1 \%$; unilateral misses $=2 \%, S E M= \pm 1 \%)$. The mean false alarm rate was $1 \%(S E M= \pm 1 \%)$.

For unilaterally presented targets, there was no difference in repetition detection between the LVF and the RVF. For bilaterally presented colors, repetitions that occurred in the upper visual field were detected $55 \mathrm{msec}$ more quickly than those in the lower visual field $[F(1,7)=$ $21.66, p<.002$, two-tailed]. Due to the significant difference in repetition detection for bilateral presentations, we again ran an additional comparison between the faster upper visual field and unilateral repetitions. Unilateral repetitions were detected significantly more quickly than repetitions in the upper visual field $[31 \mathrm{msec} ; F(1,7)=$ $15.05 ; p<.006$, two-tailed].

\section{Discussion}

A unilateral field advantage was found for detecting repeated colors, indicating that the effect is not limited to letter stimuli. The 56-msec benefit for detecting unilateral repetitions (Figure 6) was smaller than the benefit in the letter tasks, but the average RT was shorter as well, so the effect was similar, relative to mean RT. For the category of bilateral presentations, we once again found that repetition detection was more rapid in the upper visual field. However, unilateral detection was again significantly faster than detection in the upper visual field, the faster of the bilateral positions.

\section{EXPERIMENT 3 Size Repetition Search}

\section{Stimuli and Procedure}

The size stimuli were filled black circles. Sizes for each trial were randomly selected from a pool of six circles with diameters of 0.25 , $0.5,1,2,3$, and $4 \mathrm{~cm}$. The size stimuli were presented at the verti- ces of an invisible $5^{\circ} \times 5^{\circ}$ square around fixation and remained on screen until the subjects gave a response. The subjects preformed 20 practice and 430 real trials. On the $50 \%$ of the trials that had a repetition, two of the four circles were matched in size.

\section{Results}

Figure 7 indicates mean RT as a function of whether the repeated size target was presented unilaterally or bilaterally to the visual fields. Mean RTs for bilateral and unilateral size targets were 764 and $734 \mathrm{msec}$, respectively. The 30-msec RT advantage for unilateral presentations was significant $[F(1,7)=8.03, p<.03$, two-tailed $]$. The targetabsent mean RT was $854 \mathrm{msec}$. The overall mean error rate was $7 \%(S E M= \pm 2 \%)$, with no difference in miss rate as a function of the targets' laterality (mean bilateral misses = $6 \%, S E M= \pm 1 \%$; mean unilateral misses $=5 \%, S E M=$ $\pm 1 \%)$. The mean false alarm rate was $2 \%(S E M= \pm 1 \%)$.

For unilateral repetitions, there was no difference in RTs as a function of whether the target pair occurred in the LVF or the RVF. For bilateral targets, repetitions in the upper visual field were detected $45 \mathrm{msec}$ more quickly than those in the lower visual field $[F(1,7)=5.83, p<.05$, two-tailed]. Due to the significant difference in repetition detection for bilateral presentations, we ran an additional comparison between repetitions in the upper visual field (faster bilateral position) and unilateral repetitions. In this case, unilateral size repetitions were not detected significantly more quickly than those in the top bilateral position.

\section{Discussion}

A unilateral field advantage was found for detecting pairs of circles of matched size. The $30-\mathrm{msec}$ benefit for detecting unilateral repetitions (Figure 7) was significant but, nevertheless, considerably smaller than the benefit seen in the letter tasks or the color repetition task. Possibly, the salience of the grouping based on size is less effective 

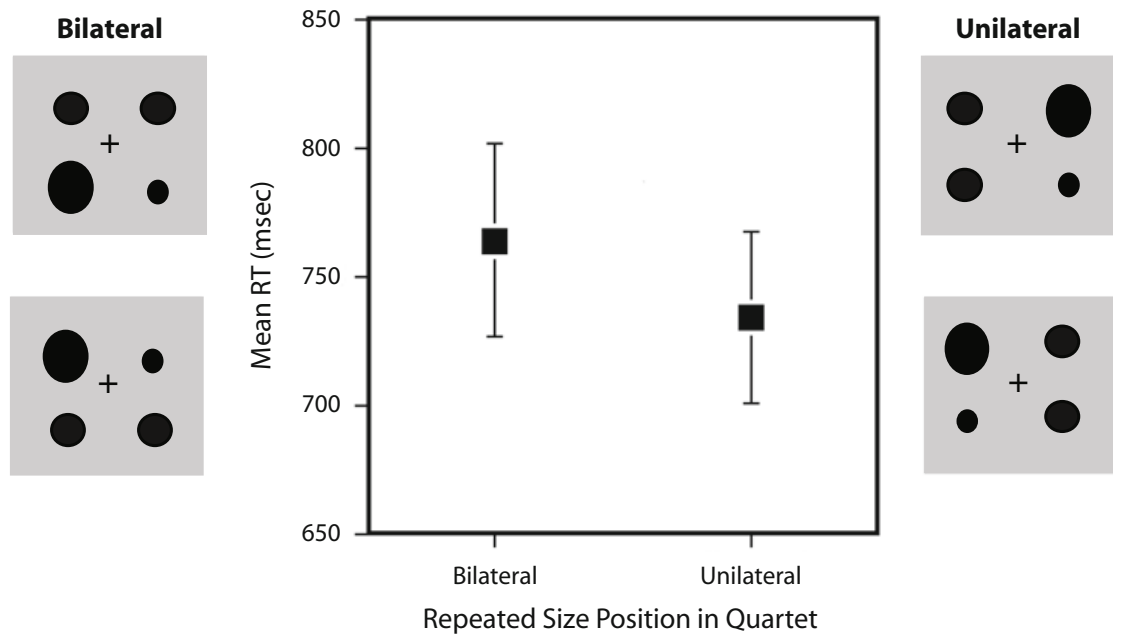

Figure 7. Experiment 3: Size repetition search. Target-present mean reaction times (RTs) for bilateral and unilaterally presented size repetitions $(n=8)$. A significant unilateral field advantage was found for detecting size repetitions.

than grouping based on color or letter physical similarity. In addition, for the category of bilateral size repetitions, the targets in the upper visual field were detected significantly more quickly than the targets in the lower visual field. In this case, there was no significant difference between unilateral repetitions and those in the upper visual field.

\section{EXPERIMENT 4 \\ Orientation Repetition Search}

\section{Stimuli and Procedure}

The stimuli for the orientation repetition search were oriented black lines that subtended $0.5 \mathrm{~cm}$ width $\times 1.5 \mathrm{~cm}$ height in vertical orientation. Orientations for each trial were selected randomly from a pool of six orientations: $0^{\circ}, 30^{\circ}, 60^{\circ}, 90^{\circ}, 120^{\circ}$, and $150^{\circ}$. The orientation stimuli were presented at the vertices of an invisible $4^{\circ} \times 4^{\circ}$ square around fixation. The subjects preformed 100 practice and 400 real trials. A repeated orientation was present on $50 \%$ of the trials.

\section{Results}

Figure 8 shows mean RT as function of the orientation repetitions' laterality. The mean RTs for bilateral and unilateral targets were 793 and $747 \mathrm{msec}$, respectively. The 45 -msec difference between these visual field presentations was not significant $[F(1,7)=3.02 ; p<.13$, twotailed]. The target-absent mean RT was $1,063.57 \mathrm{msec}$. The mean error was $7 \%(S E M= \pm 1 \%)$. There was no difference between misses as a function of the size targets' laterality (mean bilateral misses $=7 \%, S E M= \pm 1 \%$;

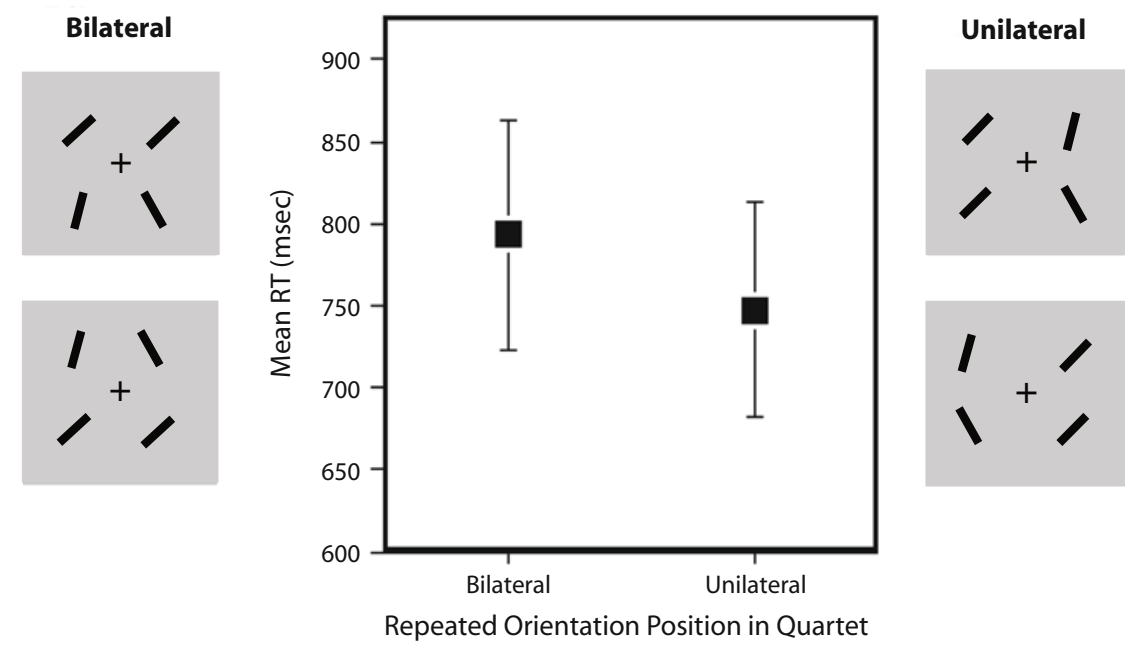

Figure 8. Experiment 4: Orientation repetition search target-present mean reaction times (RTs) as a function of the target's laterality $(n=8)$. The RT difference between bilateral and unilateral repetitions did not reach significance. 
mean unilateral misses $=6 \%, S E M= \pm 1 \%$ ). The mean false alarm rate was $2 \%(S E M= \pm 1 \%)$.

For unilateral repetitions, the difference in RT between the LVF (697 msec) and RVF (793 msec) was large but not significant, since the difference varied widely across subjects (range from 200 to $100 \mathrm{msec}$ ). For bilateral orientation repetitions, there was no difference in RTs for targets presented in the upper versus lower visual field. The mean RT was $777 \mathrm{msec}$ for the upper visual field, and $809 \mathrm{msec}$ for the lower visual field.

\section{Discussion}

There was no significant advantage in RT for detecting unilateral, as opposed to bilateral, orientation repetitions. Nevertheless, the trend in the RT data in this experiment was in the same direction as that in the previous experiments, and the numerical difference, $45 \mathrm{msec}$, fell within the range found in the previous two experiments (50 and $30 \mathrm{msec}$ ). All 8 subjects were, in fact, faster at detecting unilateral than bilateral repetitions (nonparametric binomial sign test, $p<.008$, two-tailed). The lack of a significant difference in mean RT for the orientation task may also reflect factors known to affect the processing of oriented lines. For example, there may be grouping factors for orientation that do not play a role for the other stimulus types. Specifically, the detection of oriented targets is facilitated by adjacent collinear elements and is suppressed by other spatial arrangements of items (Polat, 1999; Polat \& Sagi, 1993). This and other factors may have led to strong configural groupings between line elements that were independent of the repetition grouping we asked the subjects to report.

\section{EXPERIMENT 5 Motion Path Repetition Search}

\section{Stimuli and Procedure}

The stimuli for motion path repetition search were white $1.5^{\circ}$ disks on a uniform gray background. Each trial began with four static disks placed at the vertices of an invisible $6^{\circ} \times 6^{\circ}$ square around fixation. After a $100-\mathrm{msec}$ interval, the disks began to move at a speed of $3.25 \% \mathrm{sec}$. Each disk could move in one of the four cardinal directions (up, down, left, or right) and maintained its direction of motion for the duration of the display. The display was present for $210 \mathrm{msec}$ (14 video frames). On target-present trials, two of the four disks moved in the same direction. On target-absent trials, all four disks moved in unique directions. Eight subjects preformed 50 practice and 400 real trials. A repeated path of motion was present on $50 \%$ of the trials. The direction of the repeated motion path was selected randomly for the target-present trials.

\section{Results}

Figure 9 indicates mean RT as a function of the repeated motion paths' laterality for the remaining 7 subjects. Unilateral repeated motion paths were detected $68 \mathrm{msec}$ more quickly than bilateral repeated motion paths $[F(1,6)=$ $8.97, p<.02$, two-tailed]. The target-absent mean RT was $598 \mathrm{msec}$. The mean error rate was $16 \%(S E M= \pm 2 \%)$. There was no evidence of a speed-accuracy trade-off (bilateral misses $=8 \%, S E M= \pm 1 \%$; unilateral misses $=$ $4 \%, S E M= \pm 1 \%)$. The mean false alarm rate was $7 \%$ $(S E M= \pm 2 \%)$. For unilateral motion repetitions, there was no difference in RT for targets that appeared in the LVF versus the RVF. Likewise, for bilateral targets, there was no difference in RTs for repetitions in the upper versus the lower visual field.

\section{Discussion}

A significant unilateral field advantage for detecting repeated elements defined by common motion was found. Thus, this extends the unilateral field advantage RT benefit to targets defined by motion, in addition to static form similarity.

\section{GENERAL DISCUSSION}

Figure 10 shows an overall summary of the unilateral field advantage for repetition detection in each experiment
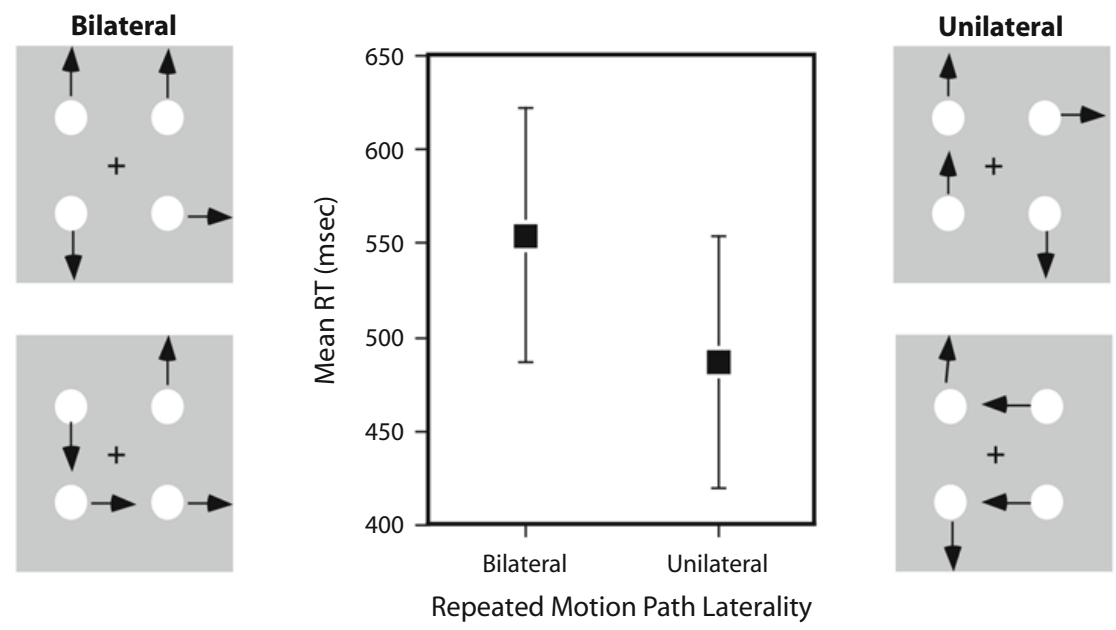

Figure 9. Common motion repetition search target-present mean reaction times (RTs) for bilateral and unilaterally presented size repetitions $(n=7)$. Unilateral repeated motion paths were detected significantly more quickly than bilateral ones $(p<.02)$. 
(with data only for the central quartet from Control Experiment 1.1). There was an RT advantage for detecting unilateral versus bilateral repetitions for the stimuli categories of letter, color, size, and common fate motion paths ranging from 30 to $112 \mathrm{msec}$. Although not significant, there was a similar RT trend for repetitions of oriented lines.

What mechanism could explain an advantage for unilateral repetition when a physical repetition is searched for? The unilateral repetitions were vertically aligned in these experiments, so the effect could have been an advantage for vertical alignment. However, the control experiment that compared vertical versus horizontal alignment when the stimuli were all within a single hemifield showed no general advantage for detecting vertically aligned repetitions.

Could the effect be a result of callosal transfer times? The bilateral repetition was detected more slowly and did require, as compared with the unilateral case, an additional transfer of information across the corpus callosum to bring the two stimuli to a common site. However, interhemispheric transfer times for detection tasks range from 2 to $3 \mathrm{msec}$ (Jeeves, 1969; Jeeves \& Dixon, 1970; Lines, Rugg, \& Milner, 1984; Ludwig et al. 1993). In contrast, the unilateral field advantage was at least $30 \mathrm{msec}$ in our experiments and as much as $112 \mathrm{msec}$. Clearly, the additional callosal transfer time can account only for a small portion of the unilateral field advantage .

Could hemispheric specialization account for the effect? Our data show no systematic difference for detecting repetitions that occurred in the LVF versus the RVF on unilateral trials. This suggests that the unilateral field advantage does not reflect hemispheric functional specificity but, rather, a general advantage for processing of information within a hemifield, left or right.

What is that processing advantage, and why do we see it here for physical repetitions, whereas the opposite effect, a bilateral advantage, is seen for letter name repetitions (Banich 1998a; Banich \& Belger, 1990; Belger \& Banich, 1992; Lavidor \& Ellis, 2003; Ludwig et al., 1993)? To answer this, we will consider two possible approaches to the task of detecting a repetition. In the first, a sequential encode-and-match process starts with one of the four display items as a target and then encodes each of the remaining three items in turn, comparing them against this first target. If no match is found, the next item is selected as the target and is compared against the remaining two items. This continues until a match is found or all pairs are compared. This sequential approach has been supported for repetition detection in visual search (Cavanagh \& Parkman, 1972), but there is no component of this process that can be invoked to give a unilateral advantage for physical repetitions but a bilateral advantage for name repetitions.

We suggest that repetition detection for a physical repetition relies, instead, on a low-level grouping process, like that seen in symmetry perception and similarity grouping. Patterns that are adjacent and physical repetitions or reflections of each other are often seen as a larger, grouped entity. If the repetition task can be based on the detection of this type of larger unit, item-by-item scrutiny, as required by the first approach, is not necessary. Moreover, since name repetitions do not support this low-level grouping, different outcomes for physical and name repetitions is not an issue.

If this low-level grouping process underlies performance in the repetition detection task, our data indicate that low-level grouping operates more efficiently within hemifields than across. This field advantage implies further that the grouping processes must be operating to some notable extent in early, retinotopic visual areas. Receptive fields in these areas are limited to the contralateral field (Abel, O'Brien, \& Olavarria, 2000; Clarke \& Miklossy, 1990; Kennedy \& Dehay, 1988), whereas in higher areas (e.g., MST, inferotemporal cortex), receptive fields often or always extend across the vertical meridian (DeYoe et al.,1996; Engel, Glover, \& Wandell, 1997; Huk, Dougherty, \& Heeger, 2002; Tootell, Mendola, Hadjikhani, Liu, \& Dale, 1998). Furthermore, this grouping process also will operate more efficiently in the periphery, since evidence suggests that foveal stimuli are represented bilaterally (Lavidor \& Walsh, 2004).

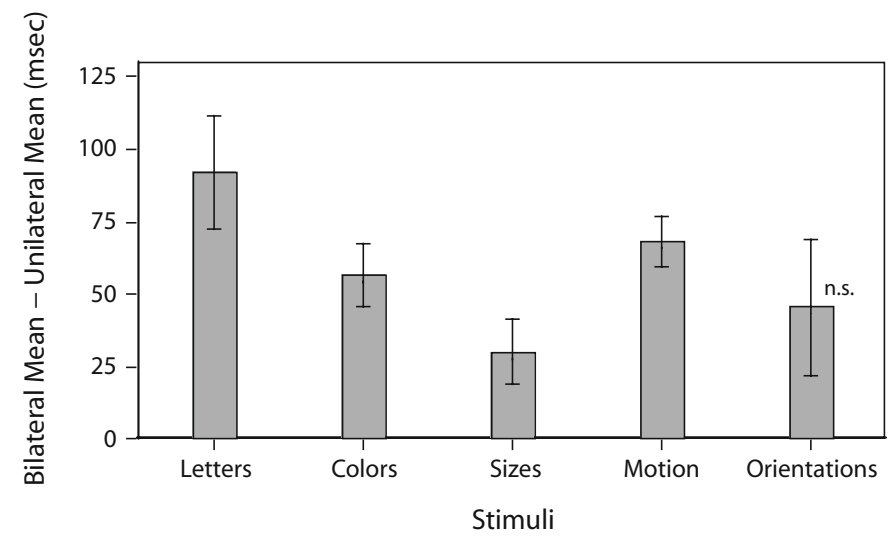

Figure 10. Summary of unilateral field advantage (bilateral mean reaction time [RT] - unilateral mean RT) for all the experiments. 
The fact that the unilateral field advantage is found for both static and moving patterns also implies that the processing benefit occurs in a low-level visual area that feeds forward to both the dorsal (what) and the ventral (where) pathways in the visual system (Ungerleider \& Mishkin, 1982). However, the data afford no more specific estimation of its neurological underpinning.

There is additional evidence that unilateral visual field presentation affords an advantage in RT when the stimulus decision involves a perceptual (vs. abstract identity) match. Banich and Belger (1990) found a within-field advantage - specifically, in the RVF - for detecting identical letters in a three-element paradigm. In their experiment, subjects were presented with two different letters around fixation (one in each hemifield), and physically matching a test letter in the lower visual field shifted toward the midline. For within-hemifield trials, the test letter matches the letter above in the same hemifield. In the betweenhemifield case, the letter match is below a nonmatching letter in the opposite hemifield. In their task, responses to RVF targets were significantly faster than responses to all other trial types.

We found, specifically, no difference for LVF and RVF letter matches and, more generally, no systematic difference between LVF and RVF targets across all the stimulus classes tested. What factors contribute to our general unilateral visual field advantage?

We suggest this may be a result of stimuli differences. In all of our experiments, four elements were presented in a square array. One possibility is that the regular spacing and bilateral symmetry of the array play an important role in producing a unilateral advantage that is not hemifield specific. It has also been suggested that equalizing hemifield processing load, as we have done here by presenting an equal number of stimuli to each hemifield, can produce within-field advantages (Weissman, Banich, \& Puente, 2000).

Further evidence in support of a unilateral field advantage for perceptual grouping of static patterns has come from studies of perceptual completion within and across hemifields. Pillow and Rubin (2002) showed that the perceptual completion of illusory contours (surface boundaries perceived in the absence of luminance gradients) was impaired when the contour crossed hemifields. In perceptual completion, the result of grouping spatially separated contours by Gestalt heuristics (e.g., good continuation and good form) is the interpolation of an illusory contour. Pillow and Rubin's data suggest an advantage for this type of grouping process when interhemispheric transfer of visual information is not required.

In addition to the unilateral field advantage, we also noted an upper field advantage in three of the six experiments (in the letter task with brief presentation, and in the color and size tasks) that ranged from 30 to $100 \mathrm{msec}$. In two of these three experiments (brief presentation letters and colors), unilateral field performance was significantly different from performance in the upper visual field, which suggests that the unilateral field RT advantage reflects a processing benefit that is distinct even from that for efficient bilateral presentations. We speculate that the upper field advantage for bilateral presentations may reflect an advantage for grouping across the hemifields when the two items are in the upper field. The spatial resolution of attention is lower (Intriligator \& Cavanagh, 2001) in the upper field, and this reduced ability to individuate adjacent targets may benefit grouping in the upper field, as compared with the lower field. This conjecture of cross-field grouping and its interaction with the resolution of spatial attention requires further testing, but the cross-field grouping, if validated, is not an alternative to the within-field grouping process that we have proposed for the unilateral field advantage; it would be a stage of grouping that naturally follows within-field grouping.

We have proposed that physical repetitions may be processed by detecting the low-level group formed by the repetition, whereas name repetitions (Banich \& Belger, 1990; Belger \& Banich, 1992; Lavidor \& Ellis, 2003; Ludwig et al., 1993) would require an item-by-item encodingand-matching process. In this view, the two tasks rely on separate mechanisms that lead to opposite effects for within- versus across-hemifield repetitions. The bilateral advantage for name match implies, as the authors of these studies have suggested, that some of the encoding processes required for naming can occur independently in each hemifield but are less efficient (perhaps even sequential) if two letters from the same hemifield must be individuated and encoded. In contrast, low-level grouping based on physical similarity treats the two patterns as one unit, and the unilateral field advantage for physical repetitions suggests that this grouping for process is more efficient within a hemifield than across hemifields.

\section{AUTHOR NOTE}

This research was supported by a Nation Science Foundation graduate fellowship to S.J.B. Correspondence concerning this article should be addressed to S. J. Butcher, Department of Psychology, Carnegie Mellon University, Baker Hall 330, 5000 Forbes Avenue, Pittsburgh, PA 15213 (e-mail: sbutcher@andrew.cmu.edu).

\section{REFERENCES}

Abel, P. L., O'Brien, B. J., \& Olavarria, J. F. (2000). Organization of callosal linkages in visual area V2 of macaque monkey. Journal of Comparative Neurology, 428, 278-293.

BANICH, M. T. (1998a). Integration of information between the cerebral hemispheres. Current Directions in Psychological Science, 7, 32-37.

BANICH, M. T. (1998b). The missing link: The role of interhemispheric interaction in attentional processing. Brain \& Cognition, 36, 128-157.

BANICH, M. T., \& BELGER, A. (1990). Interhemispheric interaction: How do the hemispheres divide and conquer a task? Cortex, 26, 77-94.

BELGER, A., \& BANICH, M. T. (1992). Interhemispheric interaction affected by computational complexity. Neuropsychologia, 30, 923-929.

Cavanagh, J. P., \& Parkman, J. M. (1972). Search processes for detecting repeated items in a visual display. Perception \& Psychophysics, 11, 43-45.

Clarke, S., \& Miklossy, J. (1990). Occipital cortex in man: Organization of callosal connections, related myelo- and cytoarchitecture, and putative boundaries of functional visual areas. Journal of Comparative Neurology, 298, 188-214.

DeYoe, E. A., Carman, G. J., Bandettini, P., Glickman, S., WieSER, J., CoX, R., ET AL. (1996). Mapping striate and extrastriate visual areas in human cerebral cortex. Proceedings of the National Academy of Sciences, 93, 2382-2386.

Engel, S. A., Glover, G. H., \& Wandell, B. A. (1997). Retinotopic organization in the human visual cortex and spatial precision of functional MRI. Cerebral Cortex, 7, 181-192. 
Huk, A. C., Dougherty, R. F., \& Heeger, D. J. (2002). Retinotopy and functional subdivisions of human areas MT and MST. Journal of Neuroscience, 22, 7195-7205.

Intriligator, J., \& CAVANAGH, P. (2001). The spatial resolution of visual attention. Cognitive Psychology, 43, 171-216.

JEeVEs, M. A. (1969). A comparison of interhemispheric transmission times in acallosals and normals. Psychonomic Science, 16, 245-246.

Jeeves, M. A., \& Dixon, N. F. (1970). Hemispheric differences in response rates to visual stimulus. Psychonomic Science, 20, 249-251.

KenNedy, H., \& Dehay, C. (1988). Functional implications of the anatomical organization of the callosal projections of visual areas V1 and V2 in the macaque monkey. Behavioural Brain Research, 29, 225-236.

LAVIDOR, M., \&, ELLIS, M. W. (2003). Interhemispheric integration of letter stimuli presented foveally or extra-foveally. Cortex, 39, 69-83.

LAVIDOR, M., \& WALSH, V. (2004). The nature of the foveal representation. National Review of Neuroscience, 5, 729-735.

Lines, C. R., RugG, M. D., \& Milner, A. D. (1984). The effect of stimulus intensity on visual evoked potential estimates of interhemispheric transfer time. Experimental Brain Research, 57, 89-98.

Ludwig, T. E., Jeeves, M. A., Norman, W. D., \&, DeWitt, R. (1993). The bilateral field advantage on a letter-matching task. Cortex, 29, 691-713.

Pillow, J., \& Rubin, N. (2002). Perceptual completion across the ver- tical meridian and the role of the early visual cortex. Neuron, $\mathbf{3 3}$, 805-813.

PoLAT, U. (1999). Functional architecture of long-range perceptual interactions. Spatial Vision, 12, 143-162.

Polat, U., \& SAGI, D. (1993). Lateral interactions between spatial channels: Suppression and facilitation revealed by lateral masking experiments. Vision Research, 33, 993-999.

Tootell, R. B. H., Mendola, J. D., Hadjikhani, N. K, LiU, A. K., \& DaLe, A. M. (1998). The representation of the ipsilateral visual field in human cerebral cortex. Proceedings of the National Academy of Sciences, 95, 818-824.

Ungerleider, L. G., \& MishKin, M. (1982). Two cortical visual systems. In D. J. Ingle, M. A. Goodale, \& R. J. W. Mansfield (Eds.), Analysis of visual behavior (pp. 549-586). Cambridge, MA: MIT Press.

WeIssman, D. H., \& Banich, M. T. (2000). The cerebral hemispheres cooperate to perform complex but not simple tasks. Neuropsychology, 14, 41-59.

Weissman, D. H., Banich, M. T., \& Puente, E. I. (2000). An unbalanced distribution of inputs across the hemispheres facilitates interhemispheric interaction. Journal of the International Neuropsychological Society, 6, 313-321.

(Manuscript received July 29, 2004;

revision accepted for publication December 18, 2007.) 